\title{
BMJ Open Differences in optimality index between planned place of birth in a birth centre and alternative planned places of birth, a nationwide prospective cohort study in The Netherlands: results of the Dutch Birth Centre Study
}

\author{
Marieke A A Hermus, ${ }^{1,2,3}$ Marit Hitzert, ${ }^{4}$ Inge C Boesveld, ${ }^{5}$ \\ M Elske van den Akker-van Marle, ${ }^{6}$ Paula van Dommelen, ${ }^{7}$ Arie Franx, ${ }^{8}$ \\ Johanna P de Graaf, ${ }^{4}$ Jan M M van Lith, ${ }^{2}$ Nathalie Luurssen-Masurel, ${ }^{1}$ \\ Eric A P Steegers, ${ }^{4}$ Therese A Wiegers, ${ }^{9}$ Karin M van der Pal-de Bruin ${ }^{1}$
}

To cite: Hermus MAA, Hitzert M, Boesveld IC, et al. Differences in optimality index between planned place of birth in a birth centre and alternative planned places of birth, a nationwide prospective cohort study in The Netherlands: results of the Dutch Birth Centre Study. BMJ Open 2017;7:e016958. doi:10.1136/ bmjopen-2017-016958

- Prepublication history for this paper is available online. To view please visit the journal online (http://dx.doi.org/10. 1136/bmjopen-2017-016958).

Received 27 March 2017 Revised 2 October 2017 Accepted 4 October 2017

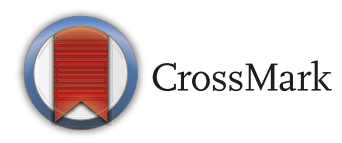

For numbered affiliations see end of article.

Correspondence to

Marieke A A Hermus; maaklapwijk@gmail.com

\section{ABSTRACT}

Objectives To compare the Optimality Index of planned birth in a birth centre with planned birth in a hospital and planned home birth for low-risk term pregnant women who start labour under the responsibility of a community midwife.

Design Prospective cohort study.

Setting Low-risk pregnant women under care of a community midwife and living in a region with one of the 21 participating Dutch birth centres or in a region with the possibility for midwife-led hospital birth. Home birth was commonly available in all regions included in the study. Participants 3455 low-risk term pregnant women (1686 nulliparous and 1769 multiparous) who gave birth between 1 July 2013 and 31 December 2013: 1668 planned birth centre births, 701 planned midwife-led hospital births and 1086 planned home births.

Main outcome measurements The Optimality Index NL-2015, a tool to measure 'maximum outcome with minimal intervention', was assessed by planned place of birth being a birth centre, a hospital setting or at home. Also, a composite maternal and perinatal adverse outcome score was calculated for the different planned places of birth.

Results There were no differences in Optimality Index NL-2015 for pregnant women who planned to give birth in a birth centre compared with women who planned to give birth in a hospital. Although effect sizes were small, women who planned to give birth at home had a higher Optimality Index NL-2015 than women who planned to give birth in a birth centre. The differences were larger for multiparous than for nulliparous women.

Conclusion The Optimality Index

NL-2015 for women with planned birth centre births was comparable with planned midwife-led hospital births. Women with planned home births had a higher Optimality Index NL-2015, that is, a higher sum score of evidence-based items with an optimal value than women with planned birth centre births.
Strengths and limitations of this study

- This is the first study to evaluate the effect of birth centre care in the Netherlands.

- The strengths of the study are the participation of all eligible Dutch birth centres (21 out of 23), the high participation rate of midwifery practices and the use of the Optimality Index NL-2015.

- The Optimality Index NL-2015 (main outcome measurement) focuses on optimality for processes and outcomes, instead of on adverse outcomes, which are rare in a population of low-risk pregnant women who are at the onset of labour under the care of a community midwife.

- Besides that the Optimality Index NL-2015 highlights a sum score of optimal events, it reflects the effect of interventions on the whole level of childbirth.

- The power of this study is too low to demonstrate a difference in adverse outcomes.

\section{INTRODUCTION}

In The Netherlands, low-risk pregnant women who start labour at or after 37 weeks gestation and are under care of a community midwife can choose whether they want to give birth at home, in a primary care level midwife-led birth centre or in the hospital. Most Dutch community midwives work in group practices with other midwives in their own premises. They are autonomous in their actions and decisions during prenatal, natal and postnatal care. ${ }^{1}$ When a complication occurs or medical assistance for pharmacological pain relief is requested, the woman will be referred to a secondary care obstetric hospital unit. Depending on the reason for 
referral, either the obstetrician or the neonatologist takes over responsibility of care from the community midwife. Reasons for referral are defined in the so-called List of Obstetric Indications. This is a multidisciplinary guideline in which all professionals involved in perinatal care have reached agreement on the indications for consultation and referral during labour and birth. ${ }^{2}$

For low-risk women who are planning to give birth out of home, there are two options, that is, in a birth centre or in a hospital setting. ${ }^{3}$ Birth centres are a relatively new development in most Dutch regions, and the number of birth centres has increased in recent years. ${ }^{45}$ Recently, a Dutch birth centre was defined as: 'a midwifery-managed location that offers care to low risk women during labour and birth. They have a homelike environment and provide facilities to support physiological birth. Community midwives take primary professional responsibility for care. In case of referral the obstetric caregiver takes over the professional responsibility of care'. ${ }^{5}$ Birth centres can be free-standing (outside the hospital), alongside (in the hospital but not in the obstetric unit) or on-site of the hospital (within the obstetric unit). The other option for low-risk women is to give birth in a conventional labour setting in a hospital room under care of a community midwife (midwife-led hospital birth). These rooms are often located in the obstetric unit and differ from the rooms in the birth centre: at this location the community midwife does not participate in the organisation of the location, protocols and birth environment. Although the community midwife is the one responsible for the care during labour and birth, this room is otherwise managed by obstetricians. In case of referral, the secondary caregiver will enter the birthing room and takes over the professional responsibility from the community midwife.

Although a woman is free to choose her preferred planned place of birth, in some occasions not all birth locations are available within her close neighbourhood, so some women have a birth centre in their neighbourhood, some a hospital and some both. In September 2013, there were 23 birth centres and 70 conventional hospital labour settings in the Netherlands. ${ }^{5}$ It is unknown what percentage of women planned birth in a birth centre or in conventional hospital labour setting because birth centres were not yet as such identified or included in the standard perinatal registration.

In The Netherlands, no research on the perinatal outcomes of planned birth centre births has been undertaken before. In other countries, studies on birth centre care have shown that low-risk women who planned to give birth in a birth centre experienced fewer interventions compared with women who planned birth in a conventional labour setting in a hospital. This included fewer intrapartum caesarean sections and lower use of obstetric analgesia and augmentation of labour. ${ }^{6-10}$ The Birthplace study in England showed that adverse perinatal outcomes (intrapartum stillbirth, early neonatal death, neonatal encephalopathy, meconium aspiration syndrome and specified birth-related injuries including brachial plexus injury) were not significantly different for low-risk nulliparous women who planned birth in free-standing midwifery units and alongside midwifery units compared with planned birth in an obstetric unit. For multiparous women, birth in free-standing and alongside midwifery units significantly and substantially reduced the odds of experiencing an unplanned caesarean section, instrumental birth or episiotomy. No significant differences in adverse perinatal outcomes were found between planned home births or midwifery unit births and planned births in obstetric units for multiparous women. ${ }^{8}$ Earlier research on the effect of planned place of birth in the Netherlands focused on the evaluation of planned birth in a conventional labour setting in a hospital and planned home birth. ${ }^{11} 12$ The national effect of planned birth in a birth centre in the Netherlands is still unknown.

In 2009, a ministerial steering committee published a report that recommended-among other thingsan investigation of the use of birth centres to improve perinatal outcomes. This was based on an assumption that birth centres might provide a higher quality of care because they offer a better opportunity for more integrated care. ${ }^{13} 14$ The essence of integrated care is a continuum of care for service users, crossing the boundaries of public health, primary, secondary and tertiary care. ${ }^{15-17}$ The increase in the number of birth centres and its unknown effect in the Dutch maternity care system, as well as the assumption that birth centres might offer more integrated care, led in 2013 to a nationwide study: the Dutch Birth Centre Study (DBC Study). The aim of that study was to evaluate birth centre care by investigating perinatal outcomes, experiences of clients and caregivers as well as economic outcomes. ${ }^{18}$ The aim of the present study, part of the DBC Study, is to assess the differences in Optimality Index NL-2015 (OI-NL2015) between a planned birth in a birth centre and a planned birth in a hospital and at home for low-risk term women who start labour under the care of a community midwife. In addition, differences in the outcomes of a planned birth in different types of birth centres based on location and level of integration were studied.

\section{METHODS}

A prospective cohort study was designed to compare the OI-NL2015 of planned birth in a birth centre with planned midwife-led hospital birth or planned home birth. Design and planning of the study were presented to the medical ethics committee of the University Medical Centre Utrecht. They confirmed that this study agrees with Dutch legal regulations for the methods used. Because of this, further formal ethical approval of this study was not required. ${ }^{19}$

\section{Setting and participants}

Within the study period 1 July 2013 to 31 December 2013, community midwives were asked to record data for each birth that started under their care regardless of 
the planned place of birth. Recruitment of the midwives was done by three researchers (MAAH, MH and ICB), two of whom are community midwives (one practising). In September 2013, there were 23 birth centres in the Netherlands according to the definition above. ${ }^{5}$ Condition for participation in this study was that the birth centre was in service for more than half a year before the start of the study period, leading to the exclusion of two birth centres. A minimum of three midwifery practices working in the area of each birth centre in the Netherlands were randomly recruited to collect data for a minimum period of 3 months. After the midwifery practice agreed on participation, the number of expected births for the next 3 months was asked to calculate the number of expected planned birth centre births. If after the recruitment of three practices this was expected to be too low, a fourth or even fifth midwifery practice was approached to participate in the study. Midwifery practices in areas where there was the possibility for midwifery-led hospital birth were randomly recruited based on their geographical location and level of urbanisation to collect data from planned midwife-led hospital births. Some midwifery practices had both options for an out-of-home birth as option for planned place of birth. Planned birth at home was an option for women in all participating midwifery practices. In total, data were obtained by 110 midwifery practices (127 were approached). In our study, 21 birth centres out of the 23 birth centres that were present in the Netherlands at that time participated as well as 46 hospital locations where midwife-led birth was possible.

Birth centres can be distinguished based on their location in relation to the obstetric unit and based on their level of integration of care. Based on location, there were three types: (1) free-standing (not on hospital grounds), (2) alongside (separate from an obstetric unit but in a hospital or on hospital grounds) or (3) on-site (within an obstetric unit of a hospital). In case of referral, physical transfer to secondary care is needed for the free-standing and alongside birth centres (response by car or ambulance or by wheelchair or bed). In case a referral is needed at the on-site birth centre, the secondary caregiver enters the birthing room of the birth centre. In the Netherlands, in September 2013, there were 3 free-standing birth centres, 14 alongside and 6 on-site birth centres. ${ }^{5}$

Boesveld et al classified birth centres into different types with distinctive characteristics. This classification was done according to their integration profile of maternity care: (1) monodisciplinary-oriented birth centres (MOBCs). MOBCs are more focused on being a facility to give birth in than on improving collaboration between maternity care providers or realising integration of care. The MOBCs are mainly owned by primary care organisations. (2) Multidisciplinary-oriented birth centres (MUBCs). MUBCs can be regarded as facilities to give birth in with a focus on integrated (birth) care. They have governance structures consisting of both primary and secondary care organisations. The disciplines involved have formulated a joint vision on birth care. The community midwife is still the person who is responsible for the care of low-risk pregnant women. (3) Birth centres with a mixed profile (MIBCs). MIBCs are a mixed group. They differ more from each other in their organisation than birth centres in the other groups. Compared with MUBCs, these centres had higher scores on clinical integration (the coordination of person-focused care in a single process across time, place and discipline) and lower scores on the other dimensions (professional, organisational, system, functional and normative integration). In September 2013, there were 10 MOBCs, 6 MUBCs and 7 MIBCs in the Netherlands. ${ }^{13}$

\section{Data collection}

In the Netherlands, individual baseline and perinatal outcome data are electronically collected in one national database: The Netherlands Perinatal Registry (Perined) ${ }^{20}$ To collect additional and more detailed data about process indicators and outcomes, a case report form (CRF) was developed for this study. ${ }^{18}$ For each pregnancy, the obtained data of the CRF were linked to data from the Netherlands Perinatal Registry by means of unique anonymous identifiers for the client and midwifery practice. Linkage between these data was obtained at the office of Perined, and the key with unique identifiers stayed there at that location, as it was proposed in the design of this study and accepted by the ethics committee. If linkage was not completed because of lacking data in Netherlands Perinatal Registry, the missing information was manually obtained from the client record in the midwifery practice and linked. Cases in which linkage between data from the CRF and data from the Netherlands Perinatal Registry was not established were excluded. Processes and outcomes were considered as non-existing if there was no registration of them in the Netherlands Perinatal Registry.

Data were collected for all term ( $\geq 37$ weeks gestational age) women at the start of labour under care of a community midwife, regardless of their planned place of birth. Excluded were women with a medium-risk situation (D-indications according to the List of Obstetric Indications, ie, an obstetric history of postpartum haemorrhage or manual removal of the placenta). ${ }^{2}$ Also, women with no specific choice for planned place of birth at the onset of labour were excluded.

Our primary main outcome measure was the OI-NL2015, a tool to measure 'maximum outcome with minimal intervention'. ${ }^{21}$ It emphasises that in general childbirth is a normal physiological process with high numbers of optimal processes and outcomes rather than a pathological process of disease. The OI-NL2015 is specifically useful to measure quality of obstetric care for women with low-risk pregnancies in which cases adverse perinatal outcomes are rare. ${ }^{22}$ The adoption of the 'optimality concept' avoids the problem of defining what is normal or abnormal in obstetrical care, and it shifts the focus from rare adverse events, that is, perinatal mortality, to evidence-based optimal events. The Optimality Index is designed to yield a summary score reflective of processes of care and clinical outcomes in relation to 
the background risk. ${ }^{21} 2324$ The OI-NL2015 has 31 items distributed over three clinical perinatal domains: intrapartum, post partum and neonatal; each item meeting the criteria for optimality is scored ' 1 '. It includes conditions (eg, pre-eclampsia) and interventions (eg, amniotomy, episiotomy, referral and epidural analgesia). Its reliability is demonstrated in earlier research. ${ }^{21}$ The OI-NL2015 is based on items that were included in the national perinatal database. The former version of a Dutch Optimality Index included a perinatal background index to adjust for differences in maternal background.$^{22}$ Because almost none of these items are included in the national perinatal database, the new version of the Optimality Index has to be adjusted, after calculating the sum score, for ethnicity, maternal age, social economic status (SES) and urbanisation level. ${ }^{21}$

Our secondary outcome measure was a description of a maternal and perinatal Composite Adverse Outcome (CAO) Score. Adverse maternal and neonatal outcomes were used to assess the effect of a planned birth in a birth centre compared with alternative settings on adverse outcomes. The CAO is a percentage based on the presence of at least one of the following adverse outcomes: maternal death (within 42 days of giving birth), third or fourth degree of perineal tear, postpartum haemorrhage ( $>1000 \mathrm{~mL}$ in 24 hours), stillbirth diagnosed after presentation in labour, early neonatal death $(<7$ days), Apgar Score $<7$ after $5 \mathrm{~min}$ and admission to a neonatal unit within 48 hours after birth. ${ }^{25}$

\section{Data analysis}

To determine whether there was a difference in Optimality Index between subgroups, the sum scores of the 31 items of the OI-NL2015 were analysed. Both outcome measures were adjusted for background variables (maternal age (mean), SES (high/medium/low), urbanisation $\left(<500\right.$ addresses per $\mathrm{km}^{2} / 500-1500$ addresses per $\mathrm{km}^{2} / \geq 1500$ addresses per $\mathrm{km}^{2}$ ) and ethnicity (Dutch/ non-Dutch)) because other studies have shown that they may vary among women with different planned places of birth and not all birth locations were available within a women's close neighbourhood. ${ }^{21}{ }^{26}$ Urbanisation and SES were based on the characteristics of the four digital postal code area in which the participants live (level of income, educational level, position in the labour market). ${ }^{27}$ Because of the large differences in interventions and outcomes between nulliparous and multiparous women, analyses were performed separately. ${ }^{28}$

To answer the research question, planned place of birth in a birth centre (reference group) was compared with planned place of birth in a hospital and home. To find out if location or level of integration of a birth centre would affect the outcome, we performed subgroup analyses between the different types of birth centres based on location and on integration level. Planned place of birth in an alongside birth centre (reference group) was compared with planned place of birth in a freestanding and an on-site birth centre. ${ }^{5}$ Planned place of birth in an MUBC (reference group) was compared with planned place of birth in MOBCs and with MIBCs. ${ }^{13}$ The sample size for this study was calculated to detect differences between the different type of birth centres on the OI-NL2015. A sample size of nine birth centres per level of integration with 66 women per centre would achieve $80 \%$ power to detect an effect size of 0.2 ( (intraclass correlation) $\mathrm{ICC}=0.005$, alpha $=0.05$ ) for the OI-NL2015 between the three levels of integration. ${ }^{11}$ Midwifery practices working with all eligible birth centres were asked to participate in this study to avoid clustering of birth centres. Based on this assumption, the power of this study would be enough to detect differences for our primary outcome measurement. All analyses were performed according to the intention-to-treat principle: data for women were analysed as belonging to the group of planned place of birth in which they were originally included.

$\chi^{2}$ tests were conducted within the nulliparous and multiparous group to compare the general characteristics and frequencies of optimality between planned places of birth. ${ }^{19}$ Logistic regression analyses were performed to adjust the frequencies of optimality and CAO Score for the general characteristics (maternal background, SES and urbanisation). Linear regression analyses were performed within the nulliparous and multiparous group to compare maternal age and the Optimality Index between all different planned places of birth. Effect sizes (Cohen's d) were calculated to examine the magnitude of the differences in OI-NL2015 between groups. It was interpreted as proposed by Cohen: small (0.2), medium (0.5) and large $(0.8) .{ }^{29}$

Although we only performed statistical tests to answer the research questions, multiple tests were performed. To take this into account, it was decided to show three levels of significance ( $\mathrm{P}$ values $<0.05,<0.005,<0.001$ ) for correct interpretation of the results.

All analyses were performed in the Statistical Package for Social Sciences V.22.0 (SPSS).

\section{Women's involvement}

Representatives of pregnant women organised in 'het Ouderschap' took place in the advisory committee of the DBC Study to advise on the set-up, planning and interpretation of the results.

Women were involved by asking for their experiences at another study that was also part of the DBC Study. ${ }^{30} \mathrm{We}$ are planning to disseminate the results of this study by means of infographics for use in the midwifery practices as a tool to inform women and their partners on the effect of planned place of birth. Results of this study will also be presented to midwives in structured peer-reviewed group sessions where the topic planned place of birth will be critically appraised.

\section{RESULTS}

After applying our exclusion criteria, 3455 women were included in the study as shown in figure 1: 1668 planned 


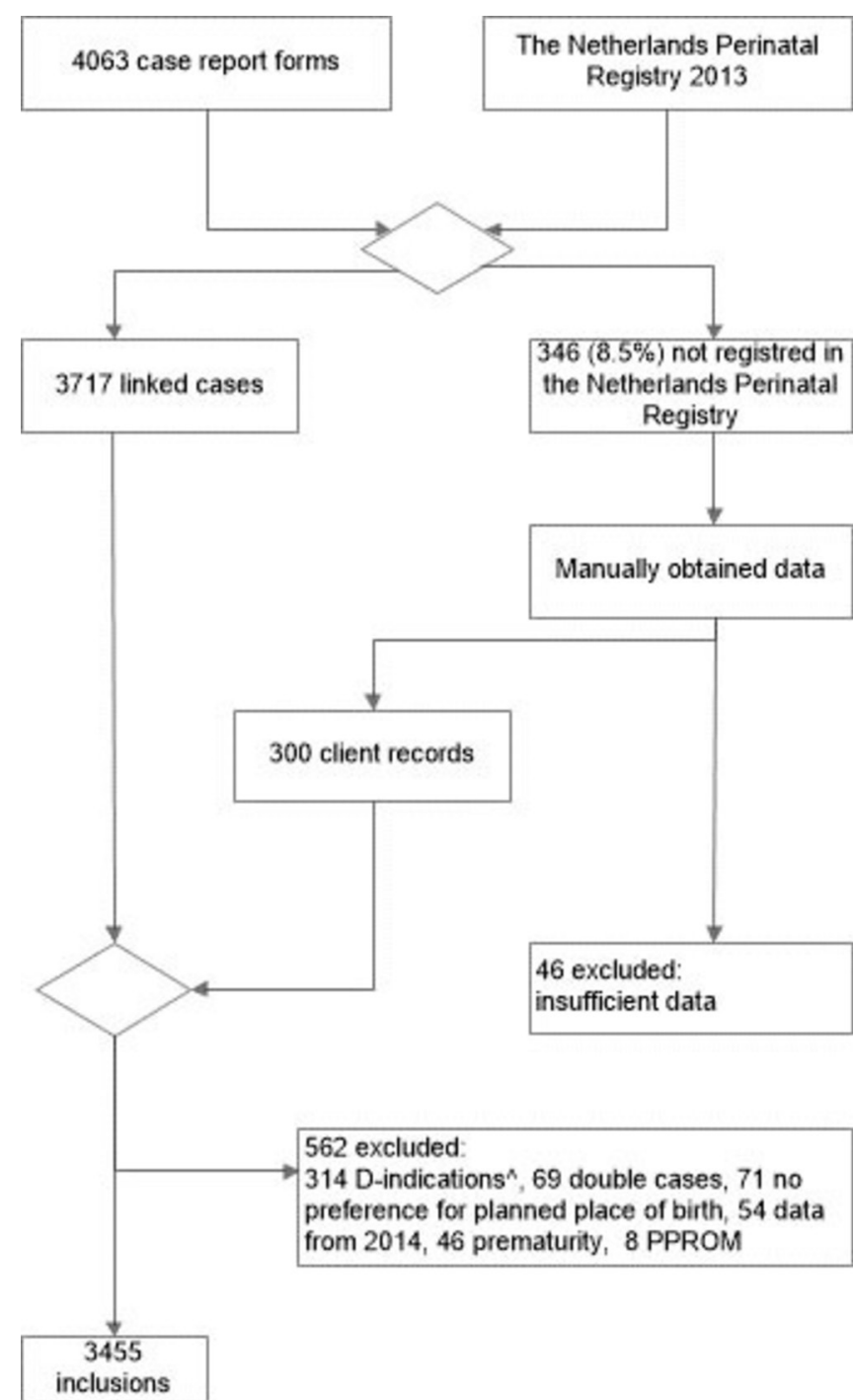

Figure 1 Flow diagram of study selection for inclusion. $\wedge \mathrm{D}$-indications according to the List of Obstetric Indications: due to medium-risk situation birth on obstetric unit $^{2}$. PPROM, premature prelabour rupture of membranes.

birth centre births, 701 planned hospital births and 1086 planned home births.

Table 1 shows the characteristics of the study population by planned place of birth. Nulliparous women who planned birth in a birth centre lived in more densely populated areas compared with nulliparous women who planned birth in a hospital $(45.0 \%$ and $30.8 \%$, respectively; $\mathrm{P}<0.05)$. Compared with women with a planned home birth, women with a planned birth centre birth were more often non-Dutch of origin, had a lower SES and lived in more densely populated areas.

\section{Individual items of the 0I-NL2015}

\section{Planned place of birth in a birth centre compared with alternative} places

The frequency of optimality for the items of the OI-NL2015 are listed in table 2 for the different planned places of birth. Interventions as epidural analgesia and episiotomy were less common in multiparous women, confirming the need to consider these women separately. For $31.8 \%$ nulliparous and $64.4 \%$ multiparous women who planned birth in a birth centre, the final place of birth was the same as the planned place of birth. Of the women who planned a midwife-led hospital or a home birth, respectively, $40.2 \%$ and $45.6 \%$ of nulliparous women and, respectively, $59.5 \%$ and $84.6 \%$ of the multiparous women succeeded in this intention.

For nulliparous women, the individual items of the OI-NL2015 demonstrated a few differences between planned place of birth in a birth centre and in a hospital, that is, "no referral during labour or within 2 hours post partum' and 'no use of oxytocin for augmentation of labour'.

For multiparous women, there were no differences in the proportion of any of the items of the OI-NL2015 between women who planned birth in a birth centre and women who planned to give birth in a hospital.

Higher proportions of optimal items were found for women who planned to give birth at home than for those who planned birth in a birth centre on the items "no referral during labour or within 2 hours post partum', 'no use of oxytocin for augmentation of labour', 'no injectable medication for pain relief during first or second stage of labour' and 'no epidural analgesia for labour and/or birth'.

\section{Location of birth centre in relation to the obstetric unit}

The final place of birth was less often in the planned place of birth for women who planned birth in an alongside birth centre (reference group) compared with women who planned birth in a free-standing birth centre (nulliparous: alongside $30.6 \%$, free-standing $69.7 \%$; multiparous: alongside $62.0 \%$, free-standing $81.3 \%$ ). Multiparous women who planned birth in an on-site birth centre were also more likely to give birth at their planned place $(71.6 \%)$ compared with the reference group $(62.0 \%)$.

For nulliparous women who planned to give birth in an alongside birth centre, 'no referral' occurred less often (29.3\%) compared with nulliparous women who planned to give birth in a free-standing birth centre $(57.6 \%)$. For multiparous women with planned birth in an alongside birth centre, "no referral 'was less common (66.2\%) compared with planned births in a free-standing birth centre $(87.5 \%)$.

'No amniotomy' and 'no episiotomy' occurred more often in women who planned to give birth in an on-site birth centre compared with women who planned to give birth in an alongside birth centre ('no amniotomy' : nulliparous: on-site $64.3 \%$, alongside $49.9 \%$; multiparous: on-site $54.6 \%$, alongside $35.0 \%$; 'no episiotomy' : nulliparous: on-site $69.6 \%$, alongside $57.7 \%$; multiparous: on-site $92.8 \%$, alongside $87.5 \%$ ). In the comparison between these two locations, the item "no manual placental removal' occurred more often for the women who planned to give birth in an alongside birth centre (nulliparous: alongside 97.7\%, on-site 94.7\%; multiparous: alongside $99.0 \%$, on-site $96.9 \%$ ). 
Table 1 Characteristics of women with low-risk pregnancies by planned place of birth at start of labour

\begin{tabular}{|c|c|c|c|c|c|c|}
\hline & \multicolumn{3}{|c|}{ Nulliparous } & \multicolumn{3}{|c|}{ Multiparous } \\
\hline & $\begin{array}{l}\text { Birth centre } \\
\text { (reference) }\end{array}$ & Hospital† & Home & $\begin{array}{l}\text { Birth centre } \\
\text { (reference) }\end{array}$ & Hospital† & Home \\
\hline & n (\%) & n (\%) & n (\%) & n (\%) & n (\%) & n (\%) \\
\hline & 939 & 348 & 399 & 729 & 353 & 687 \\
\hline \multicolumn{7}{|l|}{ Maternal background } \\
\hline Dutch & 750 (79.9) & $270(77.6)$ & $386(96.7)^{\star \star \star}$ & $524(71.9)$ & $261(73.9)$ & $649(94.5)^{\star \star \star}$ \\
\hline Non-Dutch & $189(20.1)$ & $78(22.4)$ & $13(3.3)$ & $205(28.1)$ & $92(26.1)$ & $38(5.5)$ \\
\hline \multicolumn{7}{|l|}{ Social economic status } \\
\hline Low & $123(13.3)$ & 36 (10.9) & $25(6.3)^{\star \star \star}$ & $121(17.0)$ & $44(12.9)$ & $28(4.1)^{\star \star \star}$ \\
\hline Medium & 637 (68.9) & $243(73.4)$ & 330 (83.3) & $476(67.0)$ & $239(70.3)$ & $558(81.9)$ \\
\hline High & $165(17.8)$ & $52(15.7)$ & $41(10.4)$ & $113(15.9)$ & $57(16.8)$ & $95(14.0)$ \\
\hline \multicolumn{7}{|l|}{ Urbanisation } \\
\hline $\begin{array}{l}\text { Densely populated areas } \\
\left(\geq 1500 / \mathrm{km}^{2}\right)\end{array}$ & $412(45.0)$ & $101(30.8)^{\star \star \star}$ & $80(20.4)^{\star \star \star}$ & $293(42.0)$ & $127(38.3)$ & $132(19.5)^{\star \star \star}$ \\
\hline $\begin{array}{l}\text { Intermediate density areas } \\
\left(500-1500 / \mathrm{km}^{2}\right)\end{array}$ & 215 (23.5) & 87 (26.5) & $120(30.5)$ & 188 (26.9) & $80(24.1)$ & $185(27.4)$ \\
\hline $\begin{array}{l}\text { Thinly populated areas }(<500 / \\
\left.\mathrm{km}^{2}\right)\end{array}$ & $288(31.5)$ & $140(42.7)$ & $193(49.1)$ & 217 (31.1) & $125(37.7)$ & 359 (53.1) \\
\hline $\begin{array}{l}\text { Maternal age (mean in years) } \\
\text { (SD) }\end{array}$ & $29.6(4.5)$ & $28.9(4.2)^{\star}$ & $28.3(4.2)$ & $32.2(4.3)$ & $32.1(4.0)$ & $32.4(4.6)$ \\
\hline Gestation (mean in days) (SD) & $280(7.4)$ & $279(7.3)$ & $280(7.6)$ & $281(6.4)$ & $280(6.6)$ & $281(6.8)$ \\
\hline
\end{tabular}

${ }^{\star} \mathrm{P}<0.05 ;{ }^{* \star *} \mathrm{P}<0.001$.

†Community midwife led.

No other differences were seen between the different planned locations of birth centres in relation to the obstetric unit on the items of the OI-NL2015.

Integration profiles of the birth centre

'No urgent referral' was more likely for nulliparous women who planned birth in MUBCs (the multi-disciplinary-oriented group) (95.9\%) compared with MIBCs (the mixed group) (90.9\%). Also 'blood loss $<1000 \mathrm{~mL}$ ' was less likely for women planning birth in MIBCs $(87.4 \%)$ compared with those planning birth in the other birth centres (MOBCs $94.4 \%$ (the mono-disciplinary-oriented group) and MUBCs 96.3\%)). 'Apgar Score $\geq 9$ after 5 min' was less likely in MUBCs (91.8\%) compared with MOBCs (95.6\%) for nulliparous women.

A higher proportion of women with planned birth in a birth centre within the group of MUBCs had 'no amniotomy' compared with women with planned birth in an MOBC or a birth centre from the mixed group (nulliparous: MUBCs 63.9\%, MOBCs 50.2\%, MIBCs 47.5\%; multiparous: MUBCs $53.7 \%$, MOBCs $34.2 \%$, MIBCs $38.4 \%)$.

\section{Optimality Index NL-2015}

Multiparous women had a higher mean sum score (28.3) (a more favourable outcome) on the OI-NL2015 than nulliparous women (26.0).
Birth centre compared with alternative places

As shown in table 3a, nulliparous women who planned birth in a birth centre had a lower mean score on the OI-NL2015 (25.8) compared with nulliparous women who planned birth in a hospital $(26.0, \mathrm{P}<0.05)$. The effect size of this difference was 0.07 (non-trivial). There was no significant difference between multiparous women who planned birth in a birth centre and in a hospital. Both nulliparous and multiparous women who planned birth in a birth centre had lower scores on the OI-NL2015 compared with women with the same parity that planned birth home (nulliparous: birth centre 25.8, home 26.3; $\mathrm{P}<0.005$; multiparous: birth centre 28.1, home 28.8; $\mathrm{P}<0.001)$. The effect size for this difference was 0.18 for nulliparous women (small) and 0.36 for multiparous women (small to medium).

Location of the birth centre in relation to the obstetric unit

Nulliparous women with planned place of birth in an alongside birth centre had a lower score on the OI-NL2015 than those with planned place of birth in a free-standing birth centre $(25.7$ vs $27.4, \mathrm{P}<0.005)$. The effect size of this difference was 0.64 (medium to high). Multiparous women who planned birth in an on-site birth centre had a higher score on the OI-NL2015 compared with those who planned birth in an alongside birth centre (28.4 vs $27.9, \mathrm{P}<0.05)$. The effect size of this difference was 0.24 (small). 


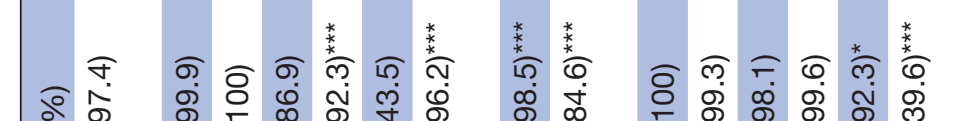

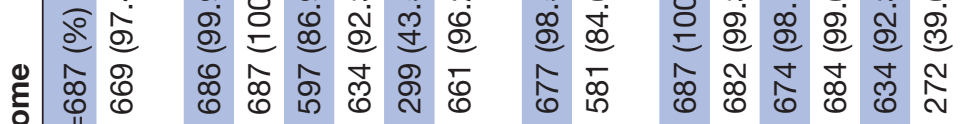

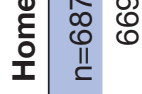

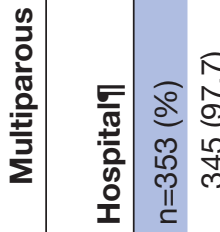

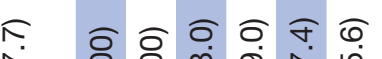

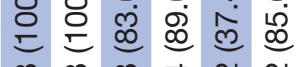

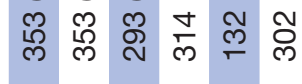

ช.

๙ั

씨 을

กิ สุ ธุ สิ

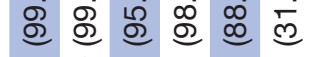

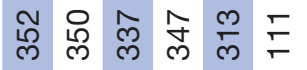

ติ

只 은

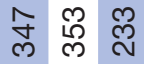

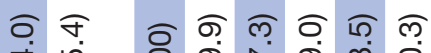

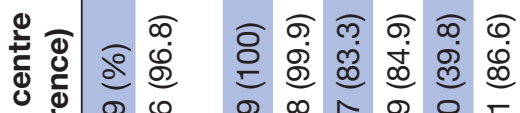

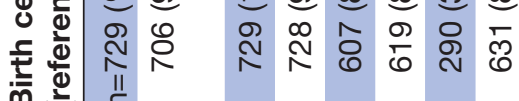

बं

点

둥 a

六 $\boldsymbol{\omega}=$

o.

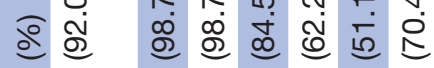

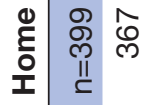

ठ্

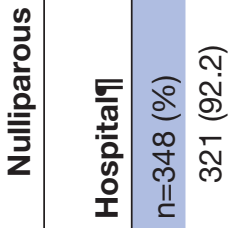

ฮุ สุ

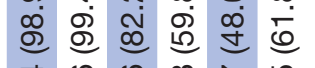

莳

హำ

लำ

ชิ ชิ

ผิ 욷

ᄃ $\cong$ व

殅

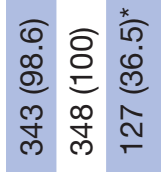

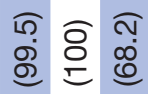

ํำ

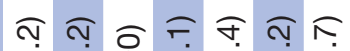

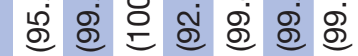

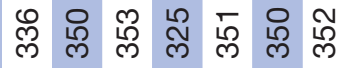

กั้

จ ธิธ

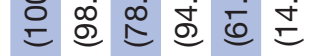

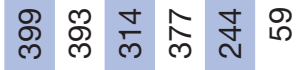

กิ

包돈

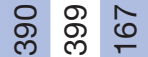

क ద

9 $\frac{8}{5}=5$

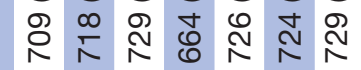

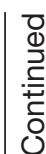

$\stackrel{\text { m }}{3}$

足

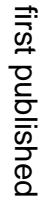

के

$\vec{\circ}$

$\overrightarrow{\vec{\omega}}$

응

옹

\&

일

$\vec{\sigma}$

zo

$\frac{\mathrm{\Phi}}{3}$

N

$\checkmark$

ำ

흥

$\frac{2}{\varnothing}$

읔

急

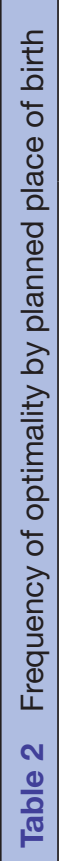

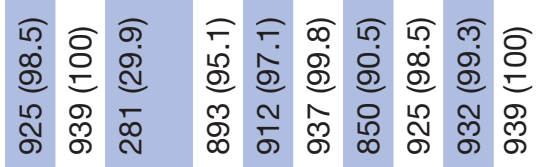

뭉

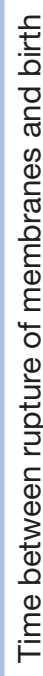

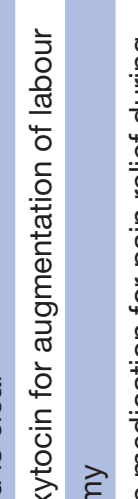

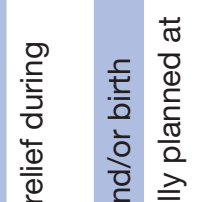

하을

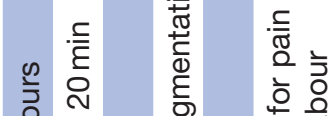

⿳亠口冋

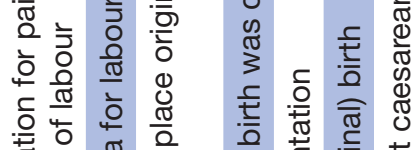

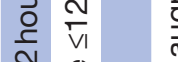

VI $\frac{0}{2} \bar{c}$

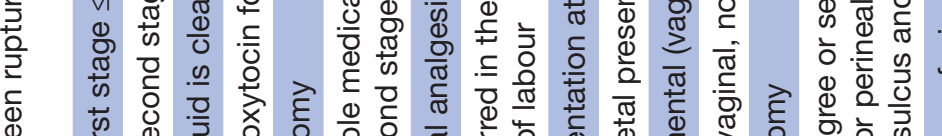

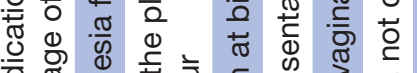

$\sum^{\infty}$ m

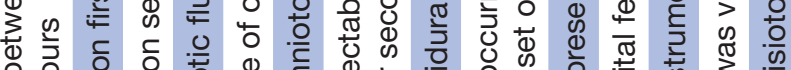

ه

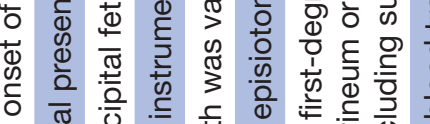

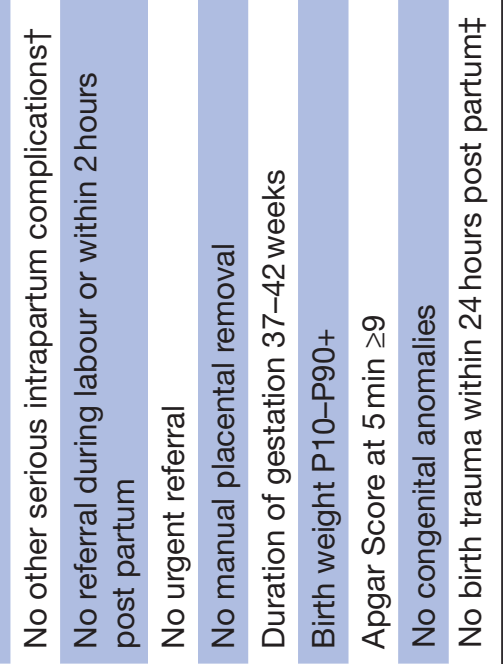




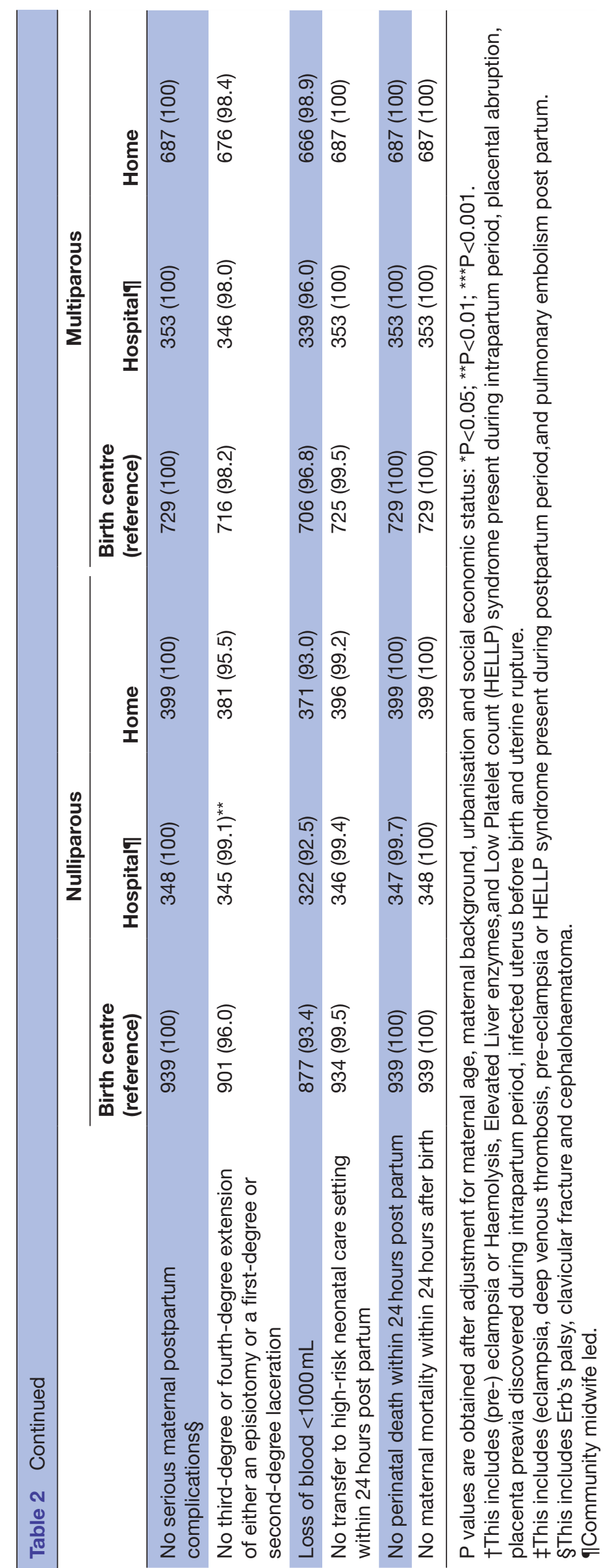

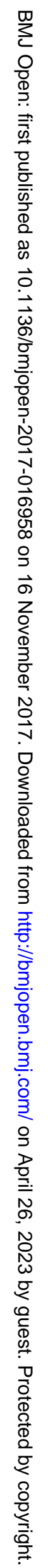


Table 3a Optimality Index NL-2015 for women with low-risk pregnancies by their planned place of birth at start of labour

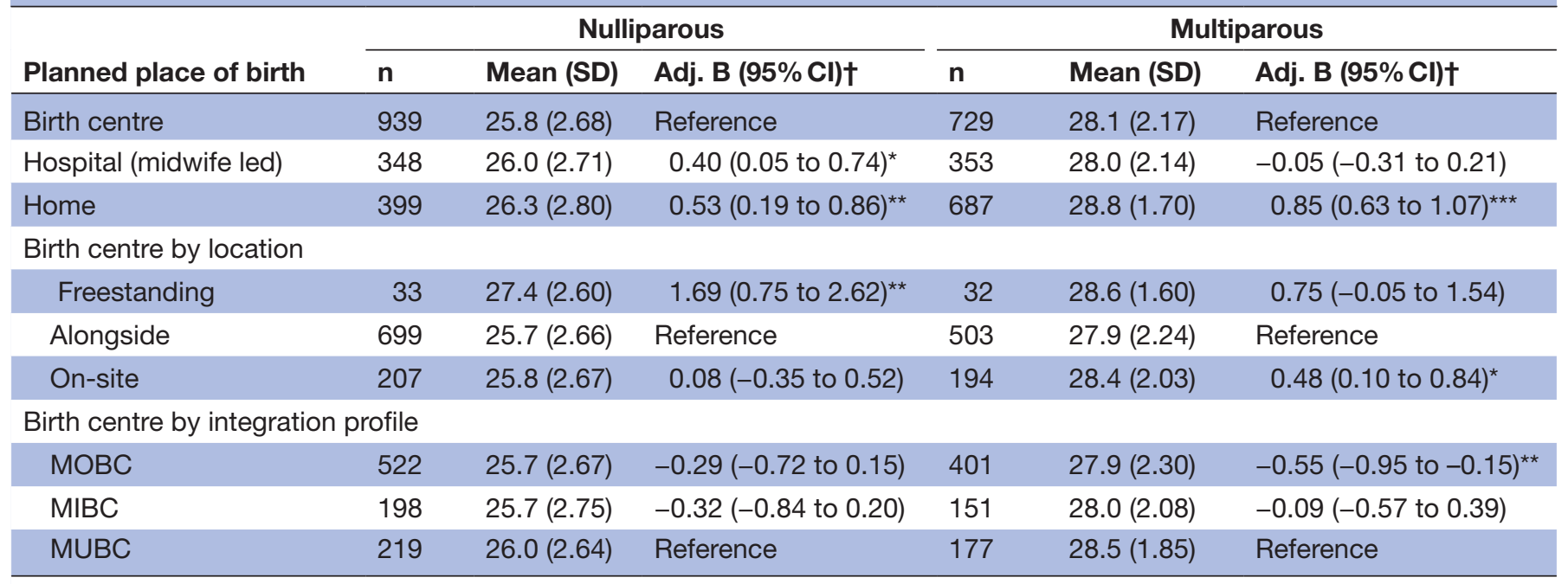

${ }^{*} \mathrm{P}<0.05 ;{ }^{* *} \mathrm{P}<0.01 ;{ }^{* * *} \mathrm{P}<0.001$

†Adjusted for maternal age, ethnicity, urbanisation and social economic status.

MIBC, birth centre with a mixed profile; MOBC, monodisciplinary-oriented birth centre; MUBC, multidisciplinary-oriented birth centre.

Integration profiles of the birth centre

For nulliparous women, no differences were found between the different types of birth centres based on their integration profile. Multiparous women who planned birth in an MUBC had a higher mean score on the OI-NL2015 compared with the women who planned birth in an MOBC (28.5 vs 27.9, $\mathrm{P}<0.005$ ). The effect size of this difference was 0.28 (small).

\section{CAO Score}

Table $3 \mathrm{~b}$ demonstrates the frequencies of the CAO between the different planned places of birth. Overall, an adverse perinatal outcome was rare. On average,

Table 3b Composite Adverse Outcome Score for women with low-risk pregnancies by their planned place of birth at start of labour

\begin{tabular}{llllll}
\hline & \multicolumn{2}{c}{ Nulliparous } & & \multicolumn{2}{c}{ Multiparous } \\
\cline { 2 - 3 } Planned place of birth & $\mathbf{n}$ & $\begin{array}{l}\text { Mean } \\
\text { (SD) }\end{array}$ & & & $\begin{array}{l}\text { Mean } \\
\text { (SD) }\end{array}$ \\
\hline Birth centre & 939 & 12.1 & 729 & 5.5 \\
Hospital (midwife led) & 348 & 10.3 & 353 & 6.2 \\
Home & 399 & 11.8 & 687 & 4.5
\end{tabular}

Birth centre by location

\begin{tabular}{lrrrr} 
Free-standing & 33 & 9.1 & 32 & 3.1 \\
\hline Alongside & 699 & 11.9 & 503 & 5.6 \\
\hline On-site & 207 & 13.5 & 194 & 5.7 \\
\hline Birth centre by integration & profile & & & \\
MOBC & 522 & 10.7 & 401 & 5.2 \\
MIBC & 198 & 18.7 & 151 & 5.3 \\
MUBC & 219 & 9.6 & 177 & 6.2 \\
\hline
\end{tabular}

MIBC, birth centre with a mixed profile; MOBC, monodisciplinaryoriented birth centre; MUBC, multidisciplinary-oriented birth centre. multiparous women had an adverse outcome less frequent than nulliparous women.

\section{DISCUSSION}

\section{Summary of main findings}

Our study demonstrated that clinically, there was no relevant difference in scores on the OI-NL2015 for women who planned to give birth in a birth centre compared with women who planned to give birth in a hospital. Only the difference between planned birth centre birth and planned home birth had a small to medium effect size: a higher score on the OI-NL2015 for women with planned home birth compared with planned birth in a birth centre.

\section{Strengths and limitations}

This was the first prospective cohort study of perinatal outcomes of planned birth in a birth centre compared with a planned birth in a hospital or at home in the Netherlands.

The OI-NL2015 focused on an evidence-based optimal approach of maternity care instead of a focus on serious adverse outcomes. Comparing groups on OI-NL2015 may show differences in processes during labour, birth and the postpartum period. Improvement of these processes could directly lead to less interventions, potentially leading to better perinatal care. Although the Optimality Index is not a commonly used outcome measure, it has been shown to be valuable over a decade in distinguishing processes of maternity care across and within various groups. ${ }^{31}$ The second approach for outcomes (CAO) is more commonly used and focused on serious adverse perinatal outcomes. ${ }^{32} 33$

Data from The Netherlands Perinatal Registry are more often used for perinatal research in the Netherlands. It 
is unclear if unregistered data in this database are not registered because they did not happen or that they are missing. In line with other research that uses these data, we considered them as not happened. It is possible that this assumption has led to a higher sum score of the OI-NL2015 (more optimal result) and an underestimation of the CAO Score.

In our study, there was an unexpected $8.5 \%$ missing data from the Netherlands Perinatal Registry. Besides a random single missing case, complete periods with data were missing from some community midwife practices. The information on missing data was shared with Perined in order to identify the cause and make it possible to solve this problem.

This study ensured comparability of the subgroups by adjusting for confounding baseline characteristics. However, women's choice for planned place of birth often reflects their underlying perception of pregnancy and childbirth. These differences have not been quantified in our previous study. ${ }^{34} 35$ Although we adjusted for common baseline characteristics, adjusting for attitude (eg, anxiety towards birth) and lifestyle (eg, smoking) was not possible in the current study. The differences in outcomes may therefore partly be a result of these confounders instead of the differences in planned location of birth.

We found that nulliparous women who planned birth in a free-standing birth centre had a higher mean score on the OI-NL2015 compared with those who planned birth in an alongside birth centre. The effect size of this difference was 0.64 (medium). Also, almost all inclusions of women with planned place of birth in a free-standing birth centre originated from one region in the Netherlands. This region is known for its conservative attitude towards healthcare in general, which may have its reflection on the perception of care of pregnant women as well as on the professional attitude of the community midwives working there. Therefore, we want to be prudent to generalise our results of planned births in a free-standing birth centre to the rest of the Dutch population. Although all women who planned birth out of home are free to choose the specific location they plan to give birth in, regional circumstances may influence their final choice, for example, facility nearest to their home available.

The enthusiastic participation of the community midwives showed the involvement and interest in this research. Their high participation rate reduced the selection bias on variation in practice among community midwives. With regard to participation of the birth centres: all eligible Dutch birth centres participated in this prospective national cohort study. The number of inclusions of planned births in the free-standing birth centres were low but in line with their annually reported low numbers of births and the number of free-standing birth centres (three) in The Netherlands.

\section{Interpretation of the results}

The difference in OI-NL2015 for women who planned birth in a birth centre compared with home was mostly due to a lower proportion of 'non-referrals'. Referral had a direct effect on the score of the individual items of the OI-NL2015, as referral often leads to the start of a cascade of interventions. ${ }^{36}$ Further analyses showed that the most important reason for this difference in number of referrals was found in referrals for failure to progress in first stage and a need for pain relief. This result was also demonstrated in earlier research on this subject. ${ }^{37}$ In July 2014, the Dutch Minister of Health, Welfare and Sports included the use of nitrous oxide as an alternative analgesia for use during labour on the list of medications to be used in primary midwifery-led care. Nitrous oxide is allowed under strict requirements for ventilation of the environment and source extraction. ${ }^{38}{ }^{39}$ It is shown to be beneficial as analgesia during labour and can be used in primary midwifery-led care in case all conditions for safety are fulfilled. ${ }^{40}$ Although it is not possible to fulfil these conditions in case of home births, birth centres can be a suitable place to offer this method for pain relief. ${ }^{29}$ Reduction of the number of referrals to secondary care could be the result.

Comparisons between birth centres distinguished by location or integration profile demonstrated that, in cases of a difference in the OI-NL2015, this was only a (very) small effect size. This effect was not homogenous across the different parities, and therefore no conclusions can be made between the different types of birth centres. A significant difference in the numbers of 'no amniotomy' was found between women with planned birth in an alongside birth centre and planned birth in an on-site birth centre. In case of meconium-stained liquor, women in an alongside birth centre need to be transferred to another room in the same hospital after referral, in contrast to women in an on-site birth centre. As it did not contribute to more referral for meconium-stained liquor, the need for amniotomy in this group should be studied in further research.

Birth centres offer facilities that may improve the chances on physiological childbirth like a birthing chair, a bath and continuous one-to-one support from a maternity care assistant. ${ }^{5}$ The actual use of these facilities and the choice of birthing position depends among other things on the perception of childbirth and the acquaintance of these facilities by the expecting woman and her partner. Also, the preferences and attitude of the attending community midwife are factors that coinfluence these choices. ${ }^{41}$ Evidence-based information about factors that make a physiological birth more or less likely should be presented antenatally to all women. The effect of the different options for planned place of birth should be included.

A clear comparison of the findings from this study to those of other birth centre studies is hard to make because the primary outcome measurement tool (OI-NL 2015) was not used before in this type of research. Other studies 
often focused on the prevalence of adverse outcomes and interventions instead of optimal outcomes. ${ }^{6-9}$ The Birthplace study in England found that women who planned birth in a midwifery unit (alongside or free-standing) had significantly fewer interventions, including substantially fewer intrapartum caesarean sections, and more spontaneous vaginal births than women who planned birth in an obstetric unit. ${ }^{6}$ That difference was not found in this study. The Birthplace study as well as this study showed that home birth is a good option for low-risk women to give birth under the care of a midwife. For women who do not want to give birth at home, birth centres are an alternative option to give birth in a home-like environment.

Personal preferences and attitude toward defining the boundaries of physiological birth may also play an important role in the use of facilities by the attending midwife to support physiological birth. In general, there is a considerable variation among this. ${ }^{42}$ Offerhaus et al showed two contrasting attitudes: (1) community midwives who 'emphasise physiology', focused on expectant management and tailor-made decisions, and (2) community midwives 'operating on the safe side', characterised by early anticipation on risks and adherence to protocols, leading to higher referral rates. As this attitude influences the whole process of care, planned place of birth is potentially coinfluenced by this. Awareness of a community midwife's personal attitude and monitoring personal referral behaviour can help to maintain highquality midwifery care. Being aware of a high referral rate can stimulate community midwives to reflect critically on what circumstances effect this rate and whether they personally can improve their care in supporting and promoting physiological childbirth, as described in the recent Lancet series. ${ }^{3643}$ A birth centre, with its home-like atmosphere and facilities to promote physiological childbirth, could be a suitable place for women who do not want to give birth at home.

\section{CONCLUSION}

This study showed that birth centres are a good alternative to give birth for the increasing number of women who do not want to give birth at home. Perinatal outcomes of planned birth centre births are comparable with planned midwife-led hospital births. Women with planned home birth had a higher OI-NL2015 compared with women with planned births in a birth centre. The pros and cons of the different places of birth should be clearly explained to women and their partners to make an informed choice on their planned place of birth.

\footnotetext{
Author affiliations

${ }^{1}$ Department of Child Health, TNO (Netherlands Organisation for Applied Scientific Research), Leiden, The Netherlands

${ }^{2}$ Department of Obstetrics, Leids Universitair Medisch Centrum, Leiden, The Netherlands

${ }^{3}$ Midwifery Practice Verloskundigen Oosterhout, Werkmansbeemd, Oosterhout, the Netherlands
}

${ }^{4}$ Department of Obstetrics and Gynaecology, Erasmus MC university Medical Centre Rotterdam, Rotterdam, The Netherlands

${ }^{5}$ Jan van Es Instituut, Almere, Flevoland, The Netherlands

${ }^{6}$ Department of Medical Decision Making, Leiden University Medical Centre, Leiden, The Netherlands

${ }^{7}$ Department of Life Style, TNO (NetherlandsOrganisation for Applied Scientific Research), Leiden, The Netherlands

${ }^{8}$ Division of Woman and Baby, University Medical Centre Utrecht, Utrecht, The Netherlands

${ }^{9}$ NIVEL(Netherlands Institute for Health Services Research), Utrecht, The Netherlands

Acknowledgements The authors would like to thank the participating women and community midwives for their time and effort in this research. We also would like to thank Joyce Dijs and Chantal Hukkelhoven for their support with the data of the Netherlands Perinatal Registry. Special thanks to Roger Heydanus for his expertise in scientific writing in English.

Contributors $\mathrm{MHe}, \mathrm{MHi}, \mathrm{IB}$, TW and $\mathrm{KMvdP}-\mathrm{dB}$ were involved in planning, recruitment of midwifery practises and conduction of the study. $\mathrm{MHe}, \mathrm{MHi}$, IB, TW and KvdPdB drafted the manuscript. MHe, PvD and NL-M analysed the data. MHe, MHi, IB, MEvdA-vM, PvD, AF, JPdG, JvL, NL-M, ES, TW and KMvdP-dB discussed the progress, results and implications of this study and read and approved the final manuscript.

Funding This study was funded by the Netherlands Organisation for Health Research and Development (ZonMw) (grant no 209020012).

Competing interests All authors have completed the ICMJE uniform disclosure format (www.icmje.org/coi_disclosure.pdf) and declare: "all authors had financial support from The Netherlands Organisation for Health Research and Development (ZonMw) for the submitted work; no financial relationships with any organisations that might have an interest in the submitted work in the previous three years; no other relationships or activities that could appear to have influenced the submitted work."

Ethics approval Medical Ethics Committee of the University Medical Centre Utrecht (the Netherlands).

Provenance and peer review Not commissioned; externally peer reviewed.

Data sharing statement The dataset is available after request to the corresponding author and approval of the Dutch Birth Centre Study Group.

Open Access This is an Open Access article distributed in accordance with the Creative Commons Attribution Non Commercial (CC BY-NC 4.0) license, which permits others to distribute, remix, adapt, build upon this work non-commercially, and license their derivative works on different terms, provided the original work is properly cited and the use is non-commercial. See: http://creativecommons.org/ licenses/by-nc/4.0/

(c) Article author(s) (or their employer(s) unless otherwise stated in the text of the article) 2017. All rights reserved. No commercial use is permitted unless otherwise expressly granted.

\section{REFERENCES}

1. KNOV. Midwifery in The Netherlands. 2016 http://www.knov.nl/ samenwerken/tekstpagina/489/midwifery-in-the-netherlands/ (accessed 15 Jul 2016).

2. College voor Zorgverzekeringen. Verloskundig vademecum. Eindrapport van de Commissie Verloskunde van het College voor Zorgverzekeringen Obstetrical manual Final report of the Obstetric working Group of the National Health Insurance Board CVZ. Diemen, 2003.

3. Stichting Perinatale Registratie Nederland. Perinatale Zorg in Nederland 2013. Utrecht, The Netherlands, 2014.

4. Wiegers T, de Graaf H, van der Pal K. De opkomst van geboortecentra en hun rol in de zorg. Tijdschr voor gezondheidswetenschappen 2012;90:475-8.

5. Hermus MAA, Boesveld IC, Hitzert M, et al. Defining and describing birth centres in The Netherlands - a component study of the Dutch Birth Centre Study. BMC Pregnancy Childbirth 2017;17:210.

6. Stewart M, Mccandlish R, Henderson J, et al. Review of evidence about clinical, psychosocial and economic outcomes for women with straightforward pregnancies who plan to give birth in a midwife-led 
birth centre, and outcomes for their babies. Report of a structured review of birth centre outcomes. Oxford, 2005.

7. Brocklehurst $P$, Hardy $P$, Hollowell J, et al. Perinatal and maternal outcomes by planned place of birth for healthy women with low risk pregnancies: the Birthplace in England national prospective cohort study. BMJ 2011;343:d7400.

8. Laws PJ, Tracy SK, Sullivan EA. Perinatal outcomes of women intending to give birth in birth centers in Australia. Birth 2010;37:28-36.

9. Hodnett ED, Downe S, Walsh D. Alternative versus conventional institutional settings for birth. Cochrane Database Syst Rev 2012.

10. Waldenstrom U, Nilsson C, Winbladh B. The Stockholm Birth Centre Trial: maternal and infant outcome. BJOG An Int J Obstet \&amp; Gynaecol 1997;104:410-8.

11. Wiegers TA, Keirse MJ, van der Zee J, et al. Outcome of planned home and planned hospital births in low risk pregnancies: prospective study in midwifery practices in The Netherlands. BMJ 1996;313:1309-13.

12. de Jonge A, van der Goes BY, Ravelli ACJ, et al. Perinatal mortality and morbidity in a nationwide cohort of 529688 low-risk planned home and hospital births. BJOG: An Int J Obstet Gynaecol 2009;116:1177-84.

13. Boesveld IC, Bruijnzeels MA, Hitzert M, et al. Typology of birth centres in the Netherlands using the Rainbow model of integrated care: results of the Dutch Birth Centre Study. BMC Health Serv Res 2017; $17: 426$.

14. Stuurgroep zwangerschap en geboorte. Een goed begin, veilige zorg rond zwangerschap en geboorte, 2009.

15. Goodwin N, Perri 6, Peck E, et al. Managing across diverse networks of care: lessons from other sectors. London, 2004.

16. Kodner DL. All together now: a conceptual exploration of integrated care. Healthc Q 2009;13 Spec No:6-15.

17. Valentijn PP, Boesveld IC, van der Klauw DM, et al. Towards a taxonomy for integrated care: a mixed-methods study. Int $\mathrm{J}$ Integr Care 2015;15:1-18.

18. Hermus MA, Wiegers TA, Hitzert MF, et al. The Dutch Birth Centre Study: study design of a programmatic evaluation of the effect of birth centre care in the Netherlands. BMC Pregnancy Childbirth 2015;15:148.

19. CCMO. central committee on research involving human subjects). http://www.ccmo.nl/en/ccmo-directives (accessed 8 Jul 2015).

20. Perined. Perined ondersteunt de geboortezorg in Nederland. Door samen te kijken hoe het beter kan. https://www.perined.nl/ (accessed 25 Apr 2016).

21. Hermus M, Boesveld I, van der Pal-de Bruin K, et al. Development of the Optimality Index-NL2015, an instrument to measure outcomes of maternity care. J Midwifery Womens Health 2017:580-8.

22. Wiegers TA, Keirse MJ, Berghs GA, et al. An approach to measuring quality of midwifery care. J Clin Epidemiol 1996;49:319-25.

23. Low LK, Miller J. A clinical evaluation of evidence-based maternity care using the Optimality Index. J Obstet Gynecol Neonatal Nurs 2006;35:786-93.

24. Sheridan M, Sandall J. Measuring the best outcome for the least intervention: can the Optimality Index-US be applied in the UK? Midwifery 2010;26:e9-e15.

25. Brunings $\mathrm{P}$, Laar Lvande, Galien Ovander, et al. Evaluation of an adverse outcome index for the quality of obstetric care delivered by multidisciplinary care chains. Int $J$ Integr Care 2013;13.
26. Anthony S, Buitendijk SE, Offerhaus PM, et al. Maternal factors and the probability of a planned home birth. Br J Obs Gyn 2004;112.

27. The Netherlands Institute for Social Research. First steps on the labour market, 2015. http://www.scp.nl/english/. (accessed 1 Jan 2015)

28. Bai J, Wong FW, Bauman A, et al. Parity and pregnancy outcomes. Am J Obstet Gynecol 2002;186:274-8.

29. Cohen J. Statistical power analysis for the behavioral sciences. 2nd edn. Hillsdale NJ, 1988.

30. Hitzert M, Hermus MAA, Scheerhagen M, et al. Experiences of women who planned birth in a birth centre compared to alternative planned places of birth. Results of the Dutch Birth Centre Study. Midwifery 2016;0:339-47.

31. Fullerton JT, Low LK, Shaw-Battista J, et al. Measurement of perinatal outcomes: a decade of experience and a future vision for the Optimality Index-US. Int J Childbirth 2011;1:171-8.

32. Boers KE, Vijgen SM, Bijlenga $\mathrm{D}$, et al. Induction versus expectant monitoring for intrauterine growth restriction at term: randomised equivalence trial (DIGITAT). BMJ 2010;341:c7087.

33. Koopmans CM, Bijlenga D, Groen $\mathrm{H}$, et al. Induction of labour versus expectant monitoring for gestational hypertension or mild pre-eclampsia after 36 weeks' gestation (HYPITAT): a multicentre, open-label randomised controlled trial. Lancet 2009;374:979-88.

34. Kleiverda G, Steen AM, Andersen I, et al. Place of delivery in The Netherlands: maternal motives and background variables related to preferences for home or hospital confinement. Eur J Obstet Gynecol Reprod Biol 1990;36:1-9.

35. Wiegers TA, Van der Zee J, Kerssens JJ, et al. Home birth or shortstay hospital birth in a low risk population in The Netherlands. Soc Sci Med 1998;46:1505-11.

36. Offerhaus PM, Geerts $\mathrm{C}$, de Jonge $\mathrm{A}$, et al. Variation in referrals to secondary obstetrician-led care among primary midwifery care practices in the Netherlands: a nationwide cohort study. BMC Pregnancy Childbirth 2015;15:42.

37. Offerhaus PM, Hukkelhoven CW, de Jonge A, et al. Persisting rise in referrals during labor in primary midwife-led care in The Netherlands. Birth 2013;40:192-201.

38. Schippers El. Regeling van de Minister van Volksgezondheid, Welzijn en Sport van 28 juli 2014, kenmerk 642455-123513-MEVA, houdende aanwijzing van apparatuur, geneesmiddelen, medische hulpmiddelen en middelen, behorende tot het deskundigheidsgebied van de verloskundige. Staatscourant. $2014 \mathrm{https}: / /$ zoek. officielebekendmakingen.nl/stcrt-2014-23083.html (accessed $15 \mathrm{Jul}$ 2016).

39. van der Kooy J, De Graaf JP, Kolder ZM, et al. A newly developed scavenging system for administration of nitrous oxide during labour: safe occupational use. Acta Anaesthesiol Scand 2012;56:920-5.

40. Klomp T, van Poppel M, Jones L, et al. Inhaled analgesia for pain management in labour. Cochrane Database Syst Rev 2012:CD009351.

41. de Jonge A, Rijnders MEB, van Diem MT, et al. Are there inequalities in choice of birthing position? Midwifery 2009;25:439-48.

42. Offerhaus PM, Korfker D, de Jonge A, et al. Submitted: midwives and variation in referral decisions during labour.

43. Renfrew MJ, McFadden A, Bastos $\mathrm{MH}$, et al. Midwifery and quality care: findings from a new evidence-informed framework for maternal and newborn care. Lancet 2014;384:1129-45. 